\title{
Use of the Phase-Integral Method to Determine the Reflection Properties of a Stratified Ionosphere
}

\author{
C. Altman ${ }^{1}$
}

\author{
Official Communication From D.S.I.R. Radio Research Station, Slough, Bucks, England
}

(Received October 15, 1964; revised November 23, 1964)

\begin{abstract}
A Pegasus computer has been programmed to give the absorption coefficients and virtual heights at vertical incidence for the main magneto-ionic components reflected from a stratified ionosphere, using the phase integral method. Typical absorption and virtual height curves are given for daytime ionospheric models. For high geomagnetic latitudes the $Z$-trace is shown to be one of the two least attenuated traces for daytime $E$-layer reflections at frequencies both above and below the gyrofrequency, and it is shown that under certain circumstances the $Z$-trace may appear at a lower virtual height than the $O$-trace. The limitations of the usual ray method which uses the group refractive index for determining virtual heights are discussed, and it is shown that a simple correction term may be added to the ray theory calculation of virtual heights to make it agree with the phase integral determination.
\end{abstract}

\section{Introduction}

The reflecting properties of the ionosphere have been calculated numerically by two main techniques. The most accurate is probably the 'full-wave' method in which the first order equations derived from Maxwell's equations and the ionospheric constitutive relations are solved by a step-by-step integration. The method, described by Budden [1955], Barron and Budden [1959] and others, obtains the solutions in terms of the electric and magnetic wavefields in perpendicular directions. A recent variant of this technique given by Pitteway [1964] considerably simplifies the integration procedure by obtaining solutions in terms of two independent solutions of the wave equation which are not coupled and which in many important cases are similar to the characteristic magnetoionic modes. The full-wave method is suited particularly to low-frequency analysis, whereas at higher frequencies the large number of steps required makes the integration prohibitively long. The results obtained by the full wave technique are not readily applicable to the propagation of pulses, especially to the calculation of virtual heights, since the perpendicular components in the Budden method, or the noncoupled modes in the Pitteway method, must first be 'unravelled' and reconstituted into characteristic magneto-ionic modes in order to permit the differentiation of phase with respect to frequency which yields the virtual heights of the $O$ - and $X$-traces. The difficulty is enhanced when two modes having the same polarization, e.g., the $O$ and $Z$ modes, exist simultaneously.

The second important technique, the phase integral method, has been described by Budden [1961], Cooper [1961], and others. It involves integrating the complex refractive index $n$, from ground level

${ }^{1}$ On leave of absence from the Technion, Israel Institute of Technology, Haifa, Israel. usually, up to the "height" (usually a complex quantity) where $n$ becomes zero; i.e., up to one of the reflection branch points of the complex refractive index. There will generally be two or three such reflection points, depending on whether the frequency is below or above the gyrofrequency. In addition there will be a coupling branch point at a complex height where the refractive indices for the characteristic magneto-ionic modes become equal. A contour of integration which encompasses the coupling point (in the sense that the coupling point lies between the contour and the real axis) will represent a coupling transition from one characteristic mode of polarization to another. Not all paths of integration connecting the reflection points to ground are physically significant [Budden, 1961]. In general there will be four distinct contours of integration associated with modes of reflection which will give independent traces on an ionogram - provided the absorption losses are not too high. The virtual height of reflection is deduced by evaluating the real part of $\int n d z$ over these integration paths for two adjacent frequencies, and the imaginary part of $\int n d z$ gives the overall absorption. The solutions thus give the type of information which is directly comparable with the results of pulse reflection experiments and may be used to compare the properties of theoretical models of the ionosphere with those actually observed.

\section{Theoretical Considerations}

\subsection{Appleton-Hartree Formula and the Branch Points}

The complex refractive index $n$ is given by the Appleton-Hartree formula, which is used in the form

$n^{2}=1-\frac{X(1-X-i Z)}{(1-i Z)(1-X-i Z)-Y^{2} \frac{\sin ^{2} \theta}{2} \pm \sqrt{F}}$ 
where

$$
F=\frac{Y^{4} \sin ^{4} \theta}{4}+Y^{2} \cos ^{2} \theta(1-X-i Z)^{2} .
$$

The symbols are the usual magneto-ionic parameters

$$
X=\frac{N e^{2}}{\epsilon_{0} m(2 \pi f)^{2}} \text { in rationalized units, } e \text { and }
$$

$m$ being the electronic charge and mass respectively, $f$ the wave frequency, $\epsilon_{0}$ the electric permittivity of free space, and

$N$ the number of electrons per unit volume.

$Y=\frac{f_{H}}{f}=\frac{|e| B}{m}$ where $f_{H}$ is the gyrofrequency and $B$ is the induction of the earth's magnetic field.

$Z=\frac{\nu}{2 \pi f}$ where $\nu$ is the electronic collision frequency.

$\theta$ is the inclination of the earth's magnetic field to the vertical.

In formula (2.1) the square root of the complex quantity $F$ is chosen using the computer's complex square root subroutine, so that the real part is always positive. When the plus sign is used, the wave will be called ordinary, and the minus sign will correspond to the extraordinary wave. The quantity $F,(2.2)$ may be rewritten in the form

$F=Y^{2} \cos ^{2} \theta\left[\left(Z_{c}^{2}-Z^{2}\right)+(X-1)^{2}\right.$

$$
+2 i Z(X-1)]
$$

where $Z_{c}=\frac{\Upsilon \sin ^{2} \theta}{2 \cos \theta}$.

Considering an upgoing wave in the ionosphere, initially it will be at a level where $X<1$, so that the quantity $F$, (2.3), will have a negative imaginary component and will be in the third or fourth quadrant of the complex plane. At $X=1, F$ crosses the real axis and has the sign of $\left(Z_{c}^{2}-Z^{2}\right)$. Thus if $Z_{c}>Z$ at $X=1$ then $\sqrt{F}$ changes continuously through $X=1$, and so too does the complex refractive index $n$; but if $Z_{c}<Z$ at $X=1$ then $\sqrt{F}$ jumps discontinuously from the negative imaginary to positive imaginary axis and continuity of the refractive index $n$ requires reversing the upper and lower signs as we cross the $X=1$ level. Thus an 'initial ordinary' becomes extraordinary, and vice versa.

The complex refractive index $n$ becomes zero when

$$
X=1-i Z
$$

for the ordinary wave and

$$
X=1 \pm Y-i Z
$$

for the extraordinary wave. These reflection points, labelled $R O, R X+$, and $R X$ - respectively occur at complex heights which are usually well within a wavelength of the real axis.

The ordinary and extraordinary refractive indices (and polarizations) become equal at the coupling point
$C$, at a complex height where ${ }^{2}$

$$
X=1+i\left(Z_{c}-Z\right) .
$$

The four points $R O, R X+, R X-$, and $C$ are, in general, the four branch points of the complex refractive index surface.

In order to locate the branch points in the complex plane, it is necessary that $X$ and $Z$ be known analytic functions of $z=x+i y$, the complex height. If an arbitrary ionospheric model or 'profile' is used, e.g., if $X$ and $Z$ are experimentally derived functions of the height $z$, then in order to locate the branch points in the complex plane it is necessary in each case to approximate the $X$ and $Z$ variations at real heights in the neighborhood of these points by analytic functions, and then by substitution to solve (2.4) to (2.6) for complex $z$. In general the branch point closest to the real axis is the one chosen.

\subsection{Location of the Branch Points for Exponential Variation of $X$ and $\boldsymbol{Z}$}

In the present investigation it has been assumed that $\log X$ and $\log Z$ vary linearly in the neighborhood of the branch points, so that

$$
\begin{aligned}
& X=X_{0} e^{a z} \\
& Z=Z_{0} e^{-b z}
\end{aligned}
$$

where $X_{0}$ and $Z_{0}$ are the values at $X=1$ or $X=1 \pm Y$, and the constants $a$ and $b$ are determined by measuring the slopes of the $\log X$ and $\log Z$ profiles, or of the chords across one or several tabular intervals near these points.

Substituting (2.7) and (2.8) into (2.4) and (2.5) it is found that the coordinates $\left(x_{R}, y_{R}\right)$ of the reflection points, measured from the $X=1$ or $X=1 \pm Y$ levels, are given by

$$
\begin{aligned}
x_{R}=\frac{1}{a} \log \left[\frac{\cos b y_{R}}{\cos (a+b) y_{R}}\right] \\
=\frac{1}{b} \log \left[\frac{Z_{0} \cos (a+b) y_{R}}{-X_{0} \sin a y_{R}}\right] .
\end{aligned}
$$

Here $y_{R}$ is necessarily negative and only such values of $y_{R}$ are accepted for which the arguments of the sin and $\cos$ terms are less than $\pi / 2$. Inspection of (2.9) shows that if $a$ and $b$ are positive, i.e., if the $X$ profile increases and the $Z$ profile decreases with height, then $x_{R}>0$, i.e., the reflection points will lie at slightly greater heights than the $X=1$ or $X=1 \pm Y$ levels. For $a$ positive and $b$ negative as in the $F$ region where electron-ion collisions predominate and consequently $Z$ increases with height, $x$ will often be negative.

${ }^{2}$ A second coupling point at

$$
X=1-i Z_{c}-i Z
$$

which would be located farther away from the real axis, and would hence be less significan physically, has been ignored. 
Substitution of (2.7) and (2.8) into (2.6) yields the coordinates $\left(x_{c}, y_{c}\right)$ of the coupling point, measured from the $X=1$ level, as given by

$$
\begin{aligned}
x_{c}=\frac{1}{a} \log \left[\frac{\cos b y_{c}-Z_{c} \sin b y_{c}}{\cos (a+b) y_{c}}\right] \\
=\frac{1}{b} \log \left[\frac{Z_{0} \cos (a+b) y_{c}}{Z_{c} \cos a y_{c}-\sin a y_{c}}\right] .
\end{aligned}
$$

When $Z_{c}>Z$ at $X=1$, the case of most general interest at high frequencies, the positive value of $y_{c}$ is chosen, and for $Z_{c}<Z$ at $X=1$, the negative value of $y_{c}$. In general $x_{c}$ may be positive or negative and $x_{c}$ may be greater or less than $x_{R o}$, i.e., the coupling point $C$ may be either above or below the ordinary wave reflection point $R O$.

Equations (2.9) and (2.10) may be solved numerically on a computer. Equating the expressions involving $y$ only, allows $y$ to be found by means of a suitable zero search procedure, and $x$ is then given explicitly as a function of $y$.

\subsection{Phase Integral Calculation of Absorption and Virtual Height}

Having located the main branch points, $z_{0}$, the complex refractive index must now be integrated from the starting level, usually the ground, along suitable contours up to these points and back again. The complex reflection coefficient, measured at the ground, is given by

$$
\exp \left[\frac{2 \pi i f}{c}\left(c t-2 \int_{0}^{z_{0}} n d z\right)\right]
$$

and the total absorption $A$ suffered by the wave is (in nepers)

$$
A=-\frac{4 \pi f}{c} \mathscr{I}\left(\int_{0}^{z_{0}} n d z\right) .
$$

Representing a pulse or wave packet at the ground, by an expression of the type

$$
\int B(f) \exp \left[\frac{4 \pi i}{c}\left(\frac{f_{c t}}{2}-f \int_{0}^{z_{0}} n d z\right)\right] d f
$$

we see that the phase at the ground will be stationary with respect to $f$ at a time $t=\tau$ given by

$$
\frac{c \tau}{2}=h^{\prime}=\frac{d}{d f} \mathscr{R}\left[f \int_{0}^{z_{0}} n d z\right]
$$

yielding the virtual height of reflection, $h^{\prime}$.

When $X$ and $Z$ are known analytic functions of $z$ it is frequently convenient to choose as contours of integration straight lines in the complex plane joining the origin to $z_{0}$ [Cooper, 1961]. But when $X$ and $Z$ are arbitrary tabulated functions of $z$ it is necessary to perform the integration mainly along the real axis.
A convenient path is along the real axis to $x_{0}=\mathscr{R}\left(z_{0}\right)$ and then parallel to the imaginary axis to $z_{0}=x_{0}+i y_{0}$, $X$ and $Z$ having the assumed variation in this region given by (2.7) and (2.8). This particular choice of contours has the advantage of allowing a direct comparison with simple ray theory.

Broadly speaking, the real axis integration (excluding possibly the last wavelength of contour path in the reflection region) represents the W.K.B. solution of the propagation equations. The "complex plane integration' from the real axis down to the branch point $z_{0}$, together with perhaps the last wavelength of contour path on the real axis, serves as a 'connection formula' linking the 'upgoing' to the 'downgoing' W.K.B. solutions on the real axis. This connection formula represents the asymptotic approximation to the Airy integral solution of the propagation equations near the branch point where the W.K.B. solutions break down [Budden, 1961]. The 'complex plane integration' is thus a mathematical procedure for calculating the absorption loss and phase change specific to the reflection (or coupling) process where the W.K.B. approximations are invalid.

The four main integration paths are shown schematically in figure 1 . There are two paths corresponding to the extraordinary waves ( $X$ waves) reflected at $R X+$ and $R X-$, respectively, which are important above and below the gyrofrequency, respectively. The ordinary wave ( $O$ wave) is reflected at $R O$, and the path yielding the $Z$-trace is that which proceeds from ground to the coupling point $C$ in the ordinary mode and thence to $R X+$ as extraordinary. We shall call the wave traveling along this path the $Z$-wave.

Writing the complex refractive index

$$
n=\mu-i \chi
$$

where $\mu$ and $\chi$ are the refractive and absorption indices respectively, and substituting $z_{0}=x_{0}+i y_{0}$ in (2.11), we obtain

$$
A=\frac{4 \pi f}{c}\left[\int_{0}^{x_{0}} \chi d x-\int_{x_{0}}^{x_{0}+i y_{0}} \mu d y\right]
$$

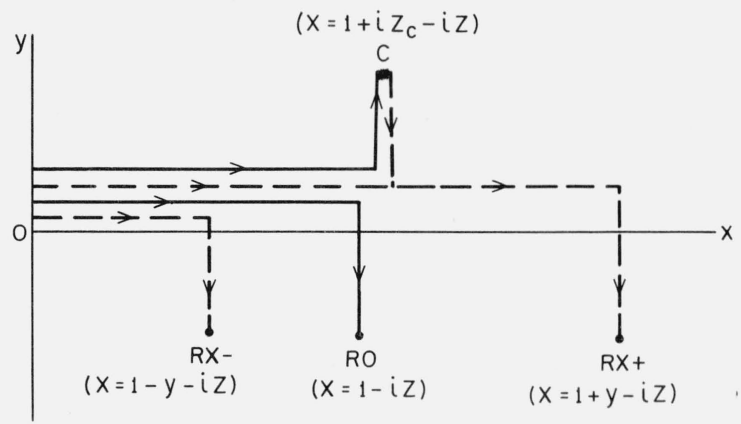

FIGURE 1. Schematic representation of the four main integration paths. 
$y_{0}$ is of course negative and the real axis and complex plane absorptions are additive. The $Z$ wave absorption may similarly be split into real axis and complex plane contributions.

The virtual height calculation (2.12) is performed by calculating

$\mathscr{R}\left[f \int_{0}^{z_{0}} n d z\right]=f\left[\int_{0}^{x_{0}} \mu d x+\int_{x_{0}}^{x_{0}+i y_{0}} \chi d y\right]$

for two nearly equal frequencies, thus giving the derivative.

\section{Results for Daytime Models}

\subsection{Ionospheric Models Used}

In order to demonstrate typical results of the computer programme two daytime ionospheric models have been chosen. In figure 2 , curve A shows the electron density distribution in the $D$ and $E$ layers over Oslo at noon during a sunspot maximum equinox. This curve has been proposed by Piggott and Thrane [1964]. Curve B shows the variation over Slough at noon during a sunspot minimum equinox. The $D$-layer curve has been determined by Deeks [1964] and the E-layer 'nose' has been tentatively fitted to it by Piggott [1964, private communication]. The inclination of the earth's field to the vertical at Oslo and Slough are $\theta=17^{\circ}$ and $\theta=22^{\circ}$, respectively, and the gyrofrequencies are $f_{H}=1.3 \mathrm{Mc} / \mathrm{s}$ and $f_{H}=1.28$ $\mathrm{Mc} / \mathrm{s}$, respectively. The dashed curve represents the mean collision frequency adopted. The two electron density distributions contrast cases with relatively dense and relatively weak $D$-region electron densities, but also differ significantly in that the slopes, $d N / d h$, are quite different for most of the reflection levels at the frequencies used in our computations.

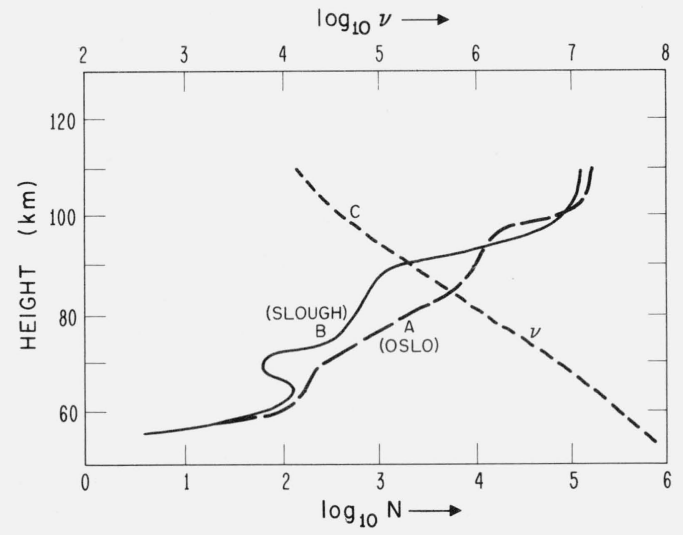

FIGURE 2. The assumed variation of electron density, N, with height at equinox noon.

Curve A is for Oslo at sunspot maximum and Curve B is for Slough, sunspot minimum. Curve $\mathrm{C}$ is the assumed height variation of electron collision frequency $\nu$.

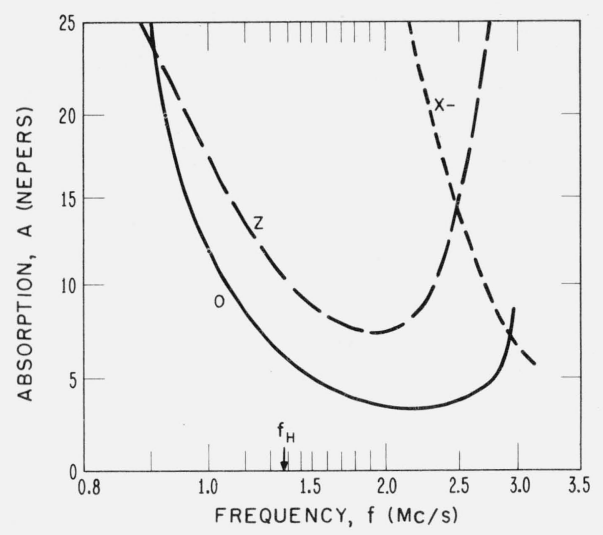

Figure 3. Absorption curves for the Oslo model. The $X+$ trace $\left(f<f_{H}\right)$ is off scale

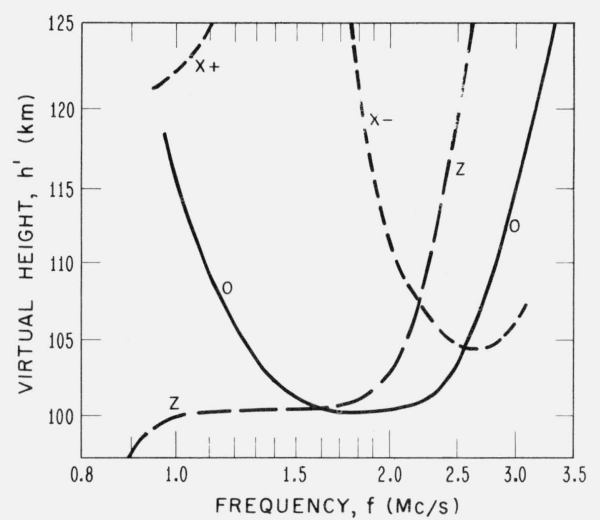

Figure 4. Virtual height curves for the Oslo model.

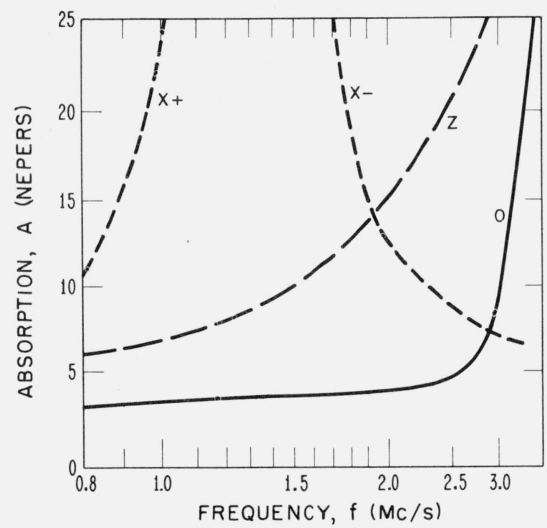

Figure 5. Absorption curves for the Slough model. 


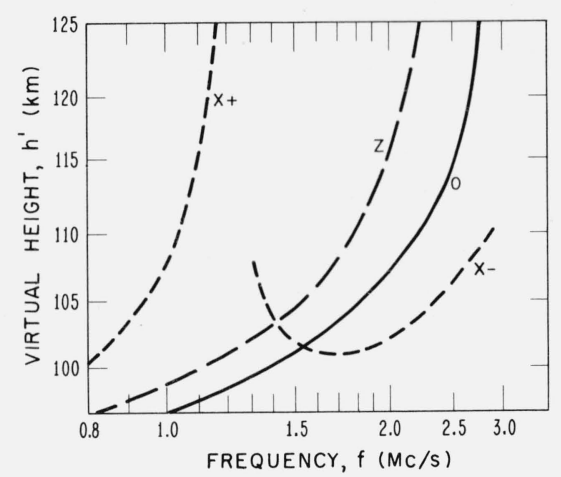

FIGURE 6. Virtual height curves for the Slough model.

\subsection{Variation With Frequency of Absorption and Virtual Heights}

Figures 3 and 4 show the absorption and virtual height variation for the Oslo model, and figures 5 and 6 repeat these for the Slough model. For all values plotted, $Z<Z_{c}$ at $X=1$. Below the gyrofrequency the $O$ - and $Z$-traces are the least attenuated, and in the case of the Oslo model the $X+$ absorption does not appear as it is off scale for frequencies above $0.9 \mathrm{Mc} / \mathrm{s}$. For both models used, an ionogram might be expected to show only an $O$-trace together with a much fainter $Z$-trace below about $2 \mathrm{Mc} / \mathrm{s}$, while an $X$-trace would only appear near the penetration frequency of the $O$-trace.

\subsection{Complex Plane Contribution to the Total Reflection Loss}

The complex plane contribution to the total absorption (given by the second term in (2.14)) has been calculated for all branch points. It never exceeds $0.05 \mathrm{~Np}$ for the $X=1+Y$ reflection in the Slough model and $0.015 \mathrm{~Np}$ in the Oslo model, and is quite negligible compared with the large real height absorption in this mode. For Oslo the $X=1-Y$ absorption in the complex plane (expressed in nepers) is less than 1 percent of the total for all frequencies above $2 \mathrm{Mc} / \mathrm{s}$, and for Slough it constitutes some 5 percent $(0.56 \mathrm{~Np})$ at $2 \mathrm{Mc} / \mathrm{s}$ falling off to about 3 percent $(0.18 \mathrm{~Np})$ at $3 \mathrm{Mc} / \mathrm{s}$. The $O$-mode complex plane absorption near $X=1$ is more significant. For Slough it constitutes $0.48 \mathrm{~Np}$ out of a total of $3.37 \mathrm{~Np}$ at $0.8 \mathrm{Mc} / \mathrm{s}$, falling off to $0.24 \mathrm{~Np}$ out of 4.31 at $2.8 \mathrm{Mc} / \mathrm{s}$. The Oslo figures are 2.67 out of $14.56 \mathrm{~Np}$ at $1 \mathrm{Mc} / \mathrm{s}$ falling to 0.26 out of $3.70 \mathrm{~Np}$ at $2.7 \mathrm{Mc} / \mathrm{s}$. The main conclusion is that the complex plane absorption is generally significant only for the $O$-wave and especially at the lower frequencies which are reflected in regions of higher collision frequency.

\subsection{Virtual Height of the $Z$-Trace}

An interesting feature of the Oslo virtual height curves is the appearance of the $Z$-trace below the
$O$-trace at the low-frequency end. This is at first sight surprising when analyzed in terms of simple ray theory, which considers the $Z$-mode wave packet to travel up to the coupling level near $X=1$ in the $O$-mode and then to travel in the $X$-mode to the $1+Y$ reflection level. The first part of the journey might be expected to contribute a virtual height equal to that of the $O$-trace, and the second part to add an additional virtual height equal to the real height difference between the $X=1$ and $X=1+Y$ levels plus any retardation in this section. The explanation of this anomalous result in the light of the phase integral analysis is given in section 4 .

\subsection{The $Z$-Trace Intensity as a Function of Magnetic Latitude}

The effect of increasing the angle of inclination $\theta$ between the earth's magnetic field and the vertical is to decrease sharply the amplitude of the $Z$-trace by increasing the coupling loss or, in terms of the phase integral, by increasing the complex plane contribution to the absorption integral, (2.11). We find, for instance, by using the Slough model distribution for a frequency of $1.1 \mathrm{Mc} / \mathrm{s}$, and letting the inclination $\theta$ take successive values, $11.5^{\circ}, 17^{\circ}, 22^{\circ}, 30^{\circ}$, and $45^{\circ}$, that the complex plane contributes an absorption loss (for two traversals, upgoing and downgoing) of 0.04, $1.2,3.0,7.1$, and $19.2 \mathrm{~Np}$ respectively. At $1.5 \mathrm{Mc} / \mathrm{s}$ the corresponding figures are $0.10,2.5,5.5,12.9$, and $30.0 \mathrm{~Np}$ respectively.

\subsection{Accuracy of the Computations}

The $O$-mode absorption calculations for the above and other ionospheric models have been checked against calculations made by a phase integral programme developed by K. G. Budden for the Cambridge Edsac II computer. Agreement is good in all cases. Slight discrepancies can be traced to the different analytic approximation made to the electron distribution near branch points. In our programme the curves are assumed to be exponential over short distances; in the Edsac II programme various other methods of curve fitting were used. This has an effect both on the computed location of branch points, and also on the interpolated values of $X$ and $Z$ between relatively widely spaced tabular intervals. The Edsac II programme checks against Budden's and against Pitteway's full wave computations.

Virtual heights for the above and other models for the ordinary and extraordinary modes have been compared with results given by a Mercury computer programme developed by E. B. Thrane in Oslo. Agreement is again good in all cases.

\section{Calculation of Virtual Heights by Simple Ray Theory and by the Phase-Integral Method}

\subsection{Phase-Integral Correction to Ray Theory}

In section 2 it was shown (2.12) that the virtual height 
of reflection is given by the expression

$$
h^{\prime}=\frac{d}{d f} \mathscr{R}\left[f \int_{0}^{\left.z_{o f}\right)} n(z) d z\right] .
$$

Since the upper limit of integration $z_{0}$ is a function of $f$ this may be written

$$
h^{\prime}=\mathscr{R}\left[\int_{0}^{z_{0}} n^{\prime} d z+f n\left(z_{0}\right) \frac{d z_{0}}{d f}\right]
$$

where

$$
n^{\prime}=\frac{d}{d f}(f n)
$$

defining the complex group refractive index.

Since $z_{0}$ represents a reflection branch point, $n\left(z_{0}\right)$ $=0$, and hence we obtain

$$
h^{\prime}=\mathscr{R} \int_{0}^{z_{0}} n^{\prime} d z
$$

Equations (4.1) and (4.4) are two equivalent phase integral expressions for the virtual height, but the method of evaluation will be different for each. Consider (4.4) first. Since $n$ and hence $n^{\prime}$ are known analytic functions of frequency, $n^{\prime}$ may be calculated for all points on the integration path and then integrated to $z_{0}$. This method is adopted by Cooper [1961]. If we compare this method with the ray theory formulation

$$
h^{\prime}=\int_{0}^{x_{0}} \mu^{\prime} d x
$$

(where $\mu^{\prime}=\mathscr{R}\left(n^{\prime}\right)$ is the group refractive index) and ignore for a moment the fact that $x_{0}$ in (4.5) is not quite equal to $\mathscr{R}\left(z_{0}\right)$ of (4.4), we find one form of the phase integral correction $\Delta h^{\prime}$, to the ray theory estimate. If $O A_{1} R_{1}$ (fig. 7) is the integration path chosen, then

$$
\Delta h^{\prime}=\mathscr{R} \int_{A_{1} R_{1}} n^{\prime} d z=\int_{A_{1} R_{1}}^{y_{0}} \quad \chi^{\prime} d y
$$

where $\chi^{\prime}$ is the group absorption index. The evaluation of this correction term necessitates doing a complex plane integration and is not readily evaluated if a phase integral computer programme is not available.

The alternative method of evaluating $h^{\prime}$ as adopted in the present investigation is based on (4.1) and gives a clearer insight into the cause of retardation, yielding also a more useful expression for the correction to ray theory. The quantity

$$
\mathscr{R}\left[f \int_{0}^{z_{0}(f)} n(z) d z\right]
$$

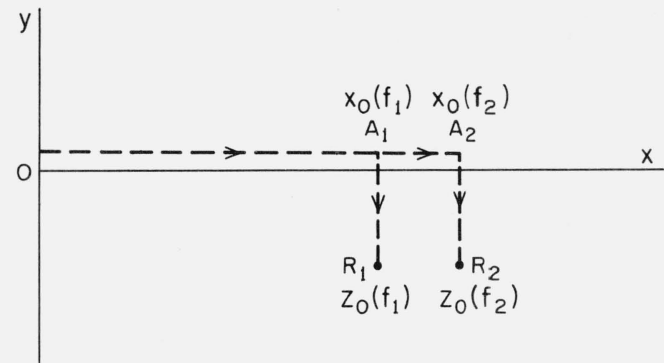

Figure 7. Contours in virtual height calculation.

$O A_{1} R_{1}$ represents an integration path to reflection point $R_{1}$ for a frequency $f_{1}$ and $O A_{2} R_{2}$ is the same for a slightly higher frequency $f_{2}$.

is evaluated for two neighboring frequencies, $f_{1}$ and $f_{2}$, the integration paths being represented schematically by $O A_{1} R_{1}$ and $O A_{2} R_{2}$, respectively (fig. 7). As $f_{1} \rightarrow f_{2}$ the virtual height is given by

$$
h^{\prime}=\Re\left[f_{2} \int_{0}^{z_{0}\left(f_{2}\right)} n d z-f_{1} \int_{0}^{z_{0}\left(f_{1}\right)} n d z\right] /\left(f_{2}-f_{1}\right)
$$

or

$$
\begin{gathered}
h^{\prime}=\left[\int_{0}^{x_{0}\left(f_{1}\right)}\left\{f_{2} \mu\left(f_{2}\right)-f_{1} \mu\left(f_{1}\right)\right\} d x+f_{2} \int_{x_{0}\left(f_{1}\right)}^{x_{0}\left(f_{2}\right)} \mu\left(f_{2}\right) d x\right. \\
\quad+\left\{f_{2} \int_{A_{2} R_{2}} \chi\left(f_{2}\right) d y\right. \\
\left.\left.-f_{1} \int_{A_{1} R_{1}} \chi\left(f_{1}\right) d y\right\}\right] /\left(f_{2}-f_{1}\right) \ldots
\end{gathered}
$$

Now the last bracketed term in the numerator representing the difference between the complex plane integrations at neighboring frequencies is usually negligible compared with the first two terms. For instance for the Slough model in the entire frequency range considered, this contribution never exceeds $0.2 \mathrm{~km}$ for the $0, X$, or $Z$ waves (unless the absorption is in excess of $100 \mathrm{~Np}$ ). Hence (4.7) in the limit reduces to

$$
h^{\prime}=\int_{0}^{x_{0}} \mu^{\prime} d x+f \mu \frac{d x_{0}}{d f}
$$

This equation may have been derived directly from (4.2) if the complex plane contribution were ignored. The second term on the right-hand side of (4.8)

$$
\Delta h^{\prime}=f \mu \frac{d x_{0}}{d f}
$$

which we shall call the 'real axis correction term' represents quite closely the difference between the phase integral and the ray method estimates of virtual height. We have thus reduced the complex plane correction to ray theory to a term involving quantities on the real axis only, the real axis coordinate $x_{0}$ of the branch point and its frequency variation being all that is required. 


\subsection{Magnitude of the 'Real Axis Correction' to Ray Theory}

Referring to (4.9) it is seen that $\Delta h^{\prime}$ is zero only when there are no collisions and $\mu\left(x_{0}\right)=0$. Its magnitude will be enhanced when $d N / d h$ is small, i.e., near a peak or in a 'ledge' of the electron density distribution. For the $E$ region $\mu\left(x_{0}\right)$ is generally appreciably larger for the $O$-wave (i.e., near $X=1$ ) than for the $X$ waves (i.e., near $X=1 \pm Y$ ) except when the latter are heavily attenuated, so that the correction term is usually more important for the $\boldsymbol{O}$-mode reflection. Neglect of this term in a true height analysis will cause overestimation of layer heights.

The term $d x_{0} / d f$ and $\Delta h^{\prime}$ have been calculated for a number of frequencies for the Oslo model, and values of $\Delta h^{\prime}$ for the $O$-mode are shown in table 1 . Values of $\mu\left(x_{0}\right)$ are also given for the $X=1$ and $X=1 \pm Y$ reflection points, labeled $R O, R X+$, and $R X-$, respectively. The quantity $\Delta x_{R}$ is the amount that $x_{0}$ lies beyond the $X=1$ level.

TABLE 1. Variation of the 'real axis correction term' $\Delta \mathrm{h}^{\prime}$ with frequency for the Oslo equinox noon value

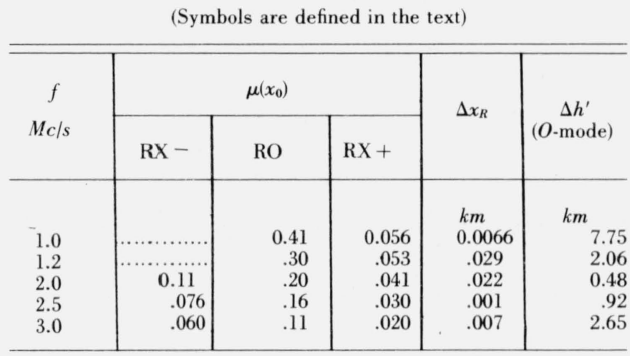

We observe that $\Delta h^{\prime}$ may be quite appreciable in the $E$ layer. It is seen that $x_{0}$ is usually very close to the ray theory reflection level $X=1$ (the same is true for the $X=1 \pm Y$ levels). This means that little error is introduced by performing the real axis integration to the $X=1$ and $X=1 \pm Y$ levels. It also means that the quantity $\frac{d x_{0}}{d f}$ appearing in the formula for $\Delta h^{\prime}$ can be approximated by calculating the frequency variation of the $X=1$ (or $X=1 \pm Y$ ) levels, which is a relatively simple procedure.

We note finally that near the peak of a noon $F_{2}$ layer, $\Delta h^{\prime}$ is of the order of $5 \mathrm{~km}$, but lower down it is very much smaller.

\subsection{Virtual Height of the Z-Trace}

The virtual height of the $Z$-trace may be calculated by similar considerations. We calculate the expression

$$
\mathscr{R}\left[f \int_{0}^{z_{0}(f)} n(z) d z\right]
$$

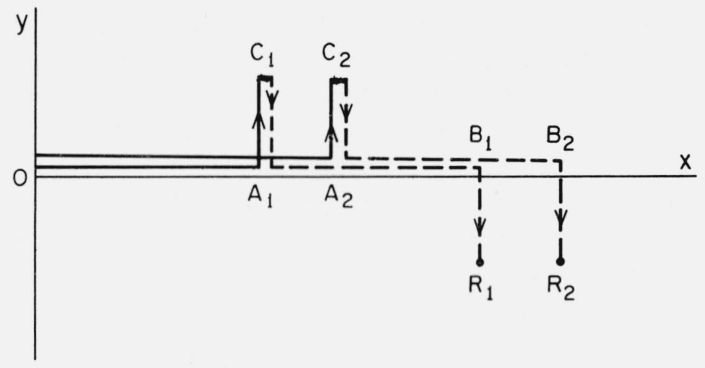

FIGURE 8. Integration contours for determining the virtual height of the Z-trace.

Key: - $O$ wave; -------- $X$ wave

for two neighboring frequencies and hence for two slightly different integration paths (fig. 8), and then calculate $h^{\prime}$ as in (4.1). This yields three main terms as in (4.7), the first corresponding to a simple ray theory calculation, the second representing a 'real axis correction term', and the third representing the difference between two pairs of complex plane integrations, which again turns out to be negligible compared with the other two terms.

The 'real axis correction term' turns out to be

$$
\left.\Delta h^{\prime}=\left[\mu_{0}(C)-\mu_{x} C\right)\right] f \frac{d x_{c}}{d f}+\mu_{x}(R) f \frac{d x_{R}}{d f}
$$

where the subscripts $o$ and $x$ refer to the ordinary and extraordinary polarizations, and $C$ and $R$ refer to the coupling and $R X+$ reflection points respectively.

The first term

$$
\left[\mu_{0}(C)-\mu_{x}(C)\right] f \frac{d x_{c}}{d f}
$$

is the real axis correction term corresponding to the coupling point integration. It is made up of two parts, the first

$$
\mu_{0}(C) f \frac{d x_{c}}{d f}
$$

being roughly equal to the usual $O$-wave real axis correction, and the second

$$
-\mu_{x}(C) f \frac{d x_{c}}{d f}
$$

whose effect is to decrease the Z-trace virtual height by this amount with respect to the O-trace. In the Oslo model at $1000 \mathrm{kc} / \mathrm{s}$ where the $O$-trace is $11 \mathrm{~km}$ above the $Z$-trace, this term is equal to $-20.5 \mathrm{~km}$ and is only partially compensated by the additional integration to the $R X+$ reflection point.

In physical terms one might say that the fact that a $Z$-trace may appear below an $O$-trace expresses the fact that the concept of a wave packet traveling to a fixed point near the $X=1$ level and there being either 
reflected, or by coupling continuing to the $R X+$ level, has only limited validity. For, in fact, each frequency component of the wave packet travels along a slightly different path and undergoes coupling or reflection at slightly different levels. This gives rise to a correction term in the virtual height determination which can produce, for instance, the appearance of a $Z$-trace below an $O$-trace.

\section{Z-Trace Both Above and Below the Gyrofrequency}

In the $F$-region $Z_{c} \gg Z$, unless $\theta \approx 0$, and the complex plane absorption loss for the $Z$-trace is usually very large. Using typical $F$-region distributions of electron density and collision frequency [Johnson, 1961] values of complex plane absorption above 100 $\mathrm{Np}$ are common, even for $\theta=17^{\circ}$. Hence the appearance of $F$-layer $Z$-traces in high latitude ionograms cannot be interpreted by means of simple magnetoionic coupling in a horizontally stratified ionosphere as described here. Budden [1961] comes to the same conclusion.

We have seen that the $E$-region $Z$-trace, which is normally ignored in theoretical analyses for frequencies below the gyrofrequency, in fact continues smoothly downwards to frequencies well below the gyrofrequency where it becomes one of the least attenuated traces. For the daytime $E$ layer when $f<f_{H}$ the $Z$-trace coupling region lies well below the $E$-layer peak and $Z$ is comparable with $Z_{c}$. As we proceed to lower frequencies the $R O$ and $C$ branch points appear at lower heights, where $Z$ is larger, and the coupling point $C$ (fig. 1) moves down until it reaches the real axis when $Z=Z_{c}$ at $X=1$. For the Slough model this would occur at $0.25 \mathrm{Mc} / \mathrm{s}$, and for Oslo at $0.8 \mathrm{Mc} / \mathrm{s}$. At this stage the upgoing ordinary becomes an upgoing extraordinary, with the coupling loss having just become zero. As $f$ is decreased further, $Z_{c}<Z$ at $X=1$, and the upgoing ordinary becomes an upgoing extraordinary at $X=1$ without coupling, and after reflection eventually reaches ground with the ordinary wave polarization. The 'initial ordinary' trace thus produced would hence be a continuation of the $Z$-trace and would usually be the strongest trace in this frequency region. Piggott, Pitteway, and Thrane [1964] have indeed found in their full-wave analysis at low frequencies that very little reflection takes place at any height besides the $X=1+Y$ level. A second, weaker trace produced by reflection at the $X=1+Y$ level would be due to the (initial) extraordinary wave, which by coupling would continue past the $X=1$ level as extraordinary, involving a discontinuity, i.e., the phase intregal contour path would have to go below the coupling point in the negative imaginary plane [Cooper, $1961]$.

Figures 9 and 10 are daytime ionograms taken at Fort Churchill $\left(\theta=6^{\circ}\right)$ showing $O$ - and $Z$-traces for the $E$ and $F$ regions. At the low frequency end of figure 9 , we see that the $Z$-trace is the only remaining trace. In figure 10 we see that for a small frequency range the $Z$-trace is unmistakably below the $O$-trace.

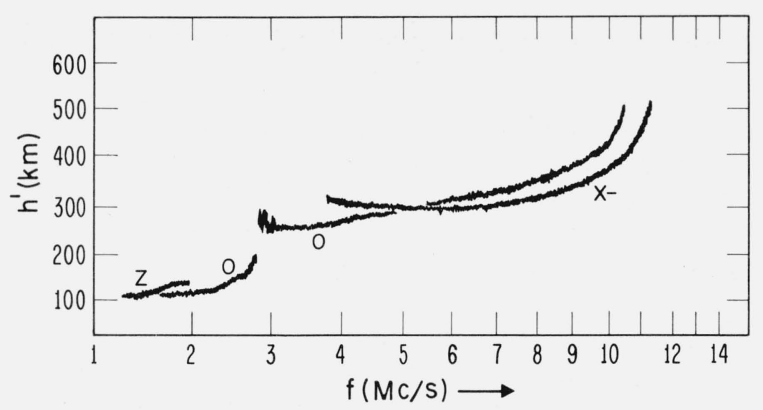

FIGURE 9. Ionogram taken at Fort Churchill at 5:30 p.m. on Sept. 13, 1958.

At the extreme low frequency end only the $Z$-trace is present.

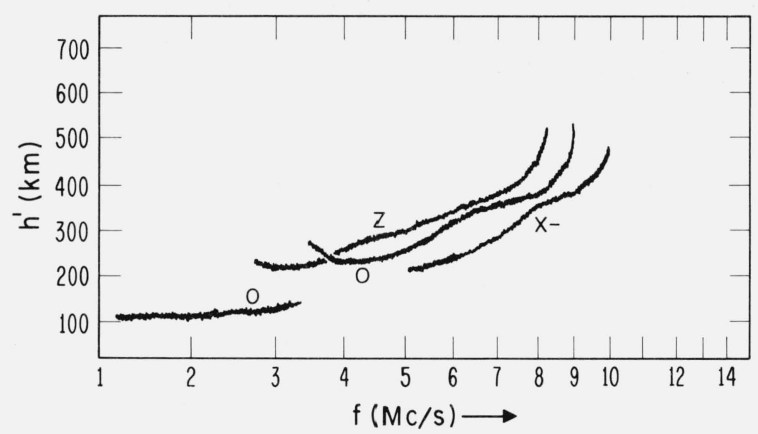

Figure 10. Ionogram taken at Fort Churchill at 10:01 a.m. on Sept. 6, 1958.

The $Z$-trace is below the $O$-trace for a small frequency range.

\section{Conclusions}

The use of a phase integral computer programme to produce absorption and virtual height versus frequency curves allows direct comparison with ionograms and pulse absorption measurements. We have shown that the $Z$-trace, which is normally ignored in the theoretical analysis for frequencies below the gyrofrequency, continues smoothly, in fact, to frequencies well below the gyrofrequency, where it becomes one of the two least attenuated traces.

It has been shown that the phase integral calculation of virtual heights can be approximated quite accurately by using the simple ray theory calculation together with a 'real axis correction term' only (4.9), requiring only the location of the branch point in the complex plane at two neighboring frequencies. In many cases this correction term can be approximated by calculating the simple ray theory reflection heights where $X=1$ and $X=1 \pm Y$, for two neighboring frequencies, together with the value of $\mu$ at these heights.

Finally it has been shown that the $Z$-trace virtual height calculation will include a term

$$
-\mu_{x}(C) f \frac{d z_{c}}{d f}
$$


which in some cases may be sufficient to produce a $Z$-trace at a lower virtual height than that of the $O$-trace.

The 'real axis correction term' is usually more significant in the $E$ region than in the $F$ region, where it is doubtful whether it would be an important factor in explaining the overestimation of heights in electron density profiles which has sometimes led to a crossing over of 'topside' and 'bottomside' $F$-region profiles.

This communication is published by kind permission of the Director of the D.S.I.R. Radio Research Station. The ionograms for Fort Churchill are published by permission of the World Data Centre for the IGY in Slough. The author is indebted to Mr. W. R. Piggott for valuable discussions in the later stages of this work, and in particular for pointing out the existence of the $E$-region $Z$-traces for high latitude stations.

(Paper 69D4-487)

\section{References}

Barron, D. W., and K. G. Budden (1959), The numerical solution of differential equations the reflection of long radio waves from the ionosphere, III, Proc. Roy. Soc. A249, 387.

Budden, K. G. (1955), The numerical solution of differential equations governing reflection of long radio waves from the ionosphere, Proc. Roy. Soc. A227, 516.

Budden, K. G. (1961), Radio waves in the ionosphere (Cambridge University Press).

Cooper, Elisabeth (1961), The properties of low frequency radio waves reflected from the ionosphere, calculated by the phase integral method, J. Atmospheric Terrest. Phys. 22, 122.

Deeks, D. G. (1964), D-region electron distributions in middle latitudes deduced from the reflection coefficients of long radio waves (private communications).

Johnson, F. S. (editor) (1961), Satellite environment handbook, pp. 28 and 40 (Stanford University Press).

Piggott, W. R., M. L. V. Pitteway, and E. V. Thrane (1964), The numerical calculation of wave-fields, reflection coefficients and polarization for long radio waves in the lower ionosphere, II, Phil. Trans. Roy. Soc. A (in press).

Piggott, W. R., and E. V. Thrane (1964), The electron densities in the $E$ - and $D$-regions deduced from studies of radio wave propagation, J. Atmospheric Terrest. Phys. (in press).

Pitteway, M. L. V. (1964), The numerical calculation of wave fields, reflection coefficients and polarizations for long radio waves in the lower ionosphere, I, Phil. Trans. Roy. Soc. A (in press). 